\title{
Efficacy and safety of ivabradine as an add-on to atenolol in patients with chronic stable ischemic heart disease
}

\author{
Elavarasi Pichai*, Ezhil Ramya J., Vasanth Sandanapitchai
}

\begin{abstract}
Department of Pharmacology, Government Thiruvarur Medical College, Tamil Nadu, India
\end{abstract}

Received: 31 August 2016 Accepted: 03 October 2016

\author{
*Correspondence to: \\ Dr. Elavarasi Pichai, \\ Email: drelavarasip@gmail.com \\ Copyright: (c) the author(s), \\ publisher and licensee Medip \\ Academy. This is an open- \\ access article distributed under \\ the terms of the Creative \\ Commons Attribution Non- \\ Commercial License, which \\ permits unrestricted non- \\ commercial use, distribution, \\ and reproduction in any \\ medium, provided the original \\ work is properly cited.
}

\begin{abstract}
Background: Heart rate is the important key factor for determining the cardiac output, myocardial oxygen demand and coronary blood flow. Reduction in heart rate is the main stay of treatment for preventing and treating IHD. Due to the interactions with other drugs and adverse effects of the conventional drugs, the management of IHD focused on novel targets. Ivabradine is a novel specific and selective if current inhibitor of SA node. The objective of the study was to efficacy and safety of ivabradine as an add-on to atenolol in patients with chronic stable ischemic heart disease.

Methods: Interventional, open label, prospective clinical study was done over a period of 1 year. Single centered study conducted in 50 patients in the Outpatient Department of cardiology, Tirunelveli Medical College Hospital, Tirunelveli. Endpoints noted were reduction in resting heart rate using 12 lead ECG and improvement in Canadian cardiovascular society (CCS) class of angina grading.

Results: Ivabradine in reducing the heart rate at the end of 1 month $(86.60 \pm 9.16)$ and at the end of 3 months $(81.82 \pm 8.37)$ compared with baseline (93.08 \pm 11.67$)$. CCS class of grade of angina also improved at the end of one month and 3 months compared with baseline. Grade IV (3 - 0 - 0), grade III (7 3 - 1) and grade II (40 - 8 - 9).

Conclusions: Ivabradine is safe and effective in preventing and treating further anginal attacks in patients with chronic stable ischemic heart disease already on atenolol therapy.
\end{abstract}

Keywords: Canadian cardiovascular society class of angina, Ivabradine, $\mathrm{I}_{\mathrm{f}}$ current, Ischemic heart disease

\section{INTRODUCTION}

Coronary heart disease is the leading cause of mortality and morbidity in developing countries and chronic stable angina pectoris is a primary symptom and the first clinical manifestation in up to $50 \%$ of patients. ${ }^{1}$ Angina pectoris is characterized by substernal discomfort, heaviness, or a pressure-like feeling, which may radiate to the jaw, shoulder, back, or arm and which typically lasts several minutes. ${ }^{2}$ Angina pectoris is caused by myocardial ischemia due to an imbalance between myocardial oxygen requirements and myocardial oxygen supply. The former may be elevated by increase in heart rate and contractility, the latter is determined by coronary blood flow and coronary arterial oxygen content. ${ }^{3}$

Heart rate is an important contributor in the pathophysiology of both coronary artery disease (CAD) and heart failure (HF). Heart rate is being recognised as a modifiable risk factor in patients with cardiovascular disease. ${ }^{4}$ Elevated heart rate increases myocardial oxygen demand and limits tissue perfusion ,the latter by shortening the duration of diastole during which most myocardial perfusion occurs. ${ }^{5}$ Pharmacotherapy for IHD is to reduce the frequency of anginal episodes, myocardial infarction, and coronary death. To achieve maximum benefit from medical therapy for IHD, it is necessary to combine agents from different classes and titrate the doses as guided by the individual profile of risk factors, symptoms, hemodynamic responses, and side effects. $^{6}$ In current clinical practice, however many patients with chronic stable IHD require treatment with more than one anti-anginal drug in addition to nitrates, beta-blockers or calcium channel blockers $(\mathrm{CCB}){ }^{7}$

Ivabradine is a novel specific and selective heart rate lowering agent that acts in sino-atrial node (SA) cells by selectively inhibiting the cardiac pacemaker in a dose dependant manner. ${ }^{8} \mathrm{I}_{\mathrm{f}}$ current has atypical or funny properties compared to other current systems such as a 
mixed $\mathrm{Na}^{+}-\mathrm{K}^{+}$inward movement activated on hyperpolarization and modulated by autonomic nervous system. It is one of the most important ionic current for regulating the pacemaker activity in the SA node. Ivabradine reduces the slope of diastolic depolarization in these cells and lowers the heart rate at rest and during exercise. $^{2,9}$

Beta blockers, unlike ivabradine, reduce $\mathrm{I}_{\mathrm{f}}$ activation by decreasing the sympathetic activity and cAMP formation, resulting in a lower HR. Left ventricular function and ventricular remodelling may be improved with $\mathrm{I}_{\mathrm{f}}$ inhibition with beta blockers like atenolol. $\mathrm{I}_{\mathrm{f}}$ inhibition with ivabradine does not alter myocardial inotropy or left ventricular function and reduces the remodelling process thus supporting cardiac output and coronary outflow during exercise. ${ }^{6}$ Beta blockers have been used only in a limited manner because of hemodynamic or pulmonary intolerance. Beta blockers should be avoided in patients with reactive airway disease (asthma) or with SA or AV nodal dysfunction or in combination with other drugs that inhibit AV conduction like verapamil. ${ }^{10}$

CCBs bind to and inhibit L-type calcium channels, reducing calcium influx into cells. Intracellular calcium deprivation relaxes smooth muscle cells, causing vasodilation in the peripheral and coronary beds and increased coronary blood flow. ${ }^{11} \mathrm{CCB}$ s can produce negative chronotropic effect thereby reduce the heart rate. However CCBs may cause reflex tachycardia, headache and edema and limit their usefulness in chronic stable angina. $^{12}$

There are still limited clinical trials related to Ivabradine and its combination with atenolol, which show its antianginal and anti-ischemic efficacy and safety in chronic stable ischemic heart disease. Thus the present study is aimed to evaluate the anti-anginal and anti-ischaemic efficacy and safety of the selective $I_{f}$ current inhibitor ivabradine in patients with chronic stable angina pectoris already receiving atenolol, beta blocker therapy.

The aim of the study was to evaluate the efficacy and safety of Ivabradine as an add-on to atenolol in patients with chronic stable ischemic heart disease prospectively.

\section{METHODS}

Interventional, open label, prospective clinical study started from April 2014 to May 2015, single centered study conducted in the Outpatient Department of Cardiology, Tirunelveli Medical College Hospital, Tirunelveli and 50 patients attending cardiology OPD receiving anti anginal medication for chronic stable ischemic heart disease.

\section{Inclusion criteria}

Patients with age of $\geq 18$ to $\leq 80$ years, both sexes with history of chronic stable ischemic heart disease, Canadian
Cardiac Society angina pectoris class 1, 2, 3 and 4,with heart rate in sinus rhythm of at least $\geq 80$ beats per minute and should be on oral tab. Atenolol for ischemic heart disease.

\section{Exclusion criteria}

Patients with age group of below 18 yrs to more than 80 yrs, heart rate of $<80$ beats per minute, $\mathrm{H} / \mathrm{O}$ of hypotension, BP $<90 / 50 \mathrm{mmhg}$ (in both the upper limbs either in the sitting or standing posture), recent or acute attack of myocardial infarction, signs and symptoms of acute decompensated heart failure, evidence of moderate to severe heart block, not taking oral tab. Atenolol for ischemic heart disease, symptomatic liver dysfunction or renal impairment, $\mathrm{H} / \mathrm{O}$ pregnancy and lactation, known hypersensitivity to study drugs and not on sinus rhythm/other types of arrhythmia, taking anti- arrhythmic drugs and taking drugs with enzyme inducing and inhibiting properties.

\section{Ethical considerations}

Approval from Institutional Ethical Committee of Tirunelveli Medical College Hospital was obtained, before starting the clinical study. Written informed consent was obtained in local vernacular language from every patient before enrolment.

\section{Methodology}

\section{Screening and recruitment}

The subjects were enrolled based on the inclusion criteria after screening. During enrolment clinical assessment and the following baseline investigations were done. Blood investigations: Hemoglobin, differential WBC count, ESR, bleeding time and clotting time were done in a blood sample using biochemical automated analyser. Blood sugar, urea and creatinine and serum electrolytes were measured in a blood sample using automated analyser. Liver function tests including ALT, AST, total bilirubin, direct and indirect bilirubin were measured in a blood sample.

Baseline heart rate was measured by taking 12 lead electrocardiography. Baseline blood pressure was measured manually after 5 minutes of rest twice at least 2 minutes apart in right arm in sitting posture with the cuff at heart level using sphygmomanometer. Baseline Canadian class of angina grading was assessed according to the clinical symptoms and signs.

\section{Treatment protocol}

After screening, Ivabradine $5 \mathrm{mg}$ once daily was prescribed to all patients who were included in this clinical study. Patient's evaluation at baseline was used as the control. The study patients were reviewed every 2 weeks. Dose of Ivabradine was titrated up to $7.5 \mathrm{mg}$ once daily according to the heart rate (if still $>100 \mathrm{bpm}$ ) after 4 
weeks of the study. Ivabradine was given orally once daily for a duration of 3 months for each patient. Also the patients were given a diary to note down the adverse events. The tablets were provided for 15 days only. Then the patients were instructed to report to the outpatient department after 2 weeks along with the diary and empty strips to collect the drugs. During each visit heart rate was monitored by taking ECG. Ivabradine tablets $5 \mathrm{mg}$ and $7.5 \mathrm{mg}$ provided by pinnacle pharmaceuticals. Compliance was assessed by reduction in heart rate and pill count methods.

\section{Follow up}

Follow up was done after 15, 30, 45, 60, 75 and 90 days, clinical examinations including vital signs such as blood pressure, heart rate were measured. At the end of $1^{\text {st }}$ and $3^{\text {rd }}$ month, laboratory investigations such as blood $\mathrm{Hb}$ gm\%, total count, ESR, serum electrolytes, LFT, blood sugar, urea and creatinine were performed. Also echocardiogram and Canadian class of angina grading based on clinical improvement in signs and symptoms was done.

\section{Efficacy parameters}

- Reduction in resting heart rate using 12 lead ECG.

- Improvement in Canadian cardiovascular society (CCS) class of angina grading.

\section{Safety assessments}

Any adverse events reported by the subject or noted by the clinician during each follow up visit was recorded .If continuation of the drug was considered harmful; the subject could be withdrawn from the study. Any adverse event was considered as serious if it was fatal, life threatening, disabling or if it prolonged hospitalization of the subject.

\section{Statistical analysis}

Statistical analysis was performed with the help of statistical package SPSS (Statistical package analysis package for the social sciences) version 11. Baseline characteristics of the study patients were tabulated by descriptive statistics (mean and standard deviation) and frequency table. The analysis of study parameters were done by using "Student paired t test" at the end of 1 and 3 months before and after giving Ivabradine. The adverse drug reactions were tabulated and expressed in percentage.

\section{RESULTS}

In the period of 1 year from April 2014 to May 2015, 60 cases of chronic stable ischemic heart disease already on tab. atenolol, attending the outpatient Department of Cardiology were screened for their eligibility based on the inclusion and exclusion criteria. Among the 60 patients, 55 patients were enrolled for the study and given tab. Ivabradine once daily orally and 5 patients were withdrawn from the study due to non-compliance and reduction in heart rate below $70 \mathrm{bpm}$. At the end of 1 month, those 50 patients continued the study and completed the study (flow chart).

Table 1: Baseline characteristics.

\begin{tabular}{|c|c|c|c|}
\hline Baseline parameters & \multicolumn{3}{|c|}{ Tab. ivabradine $(\mathrm{n}=50)$} \\
\hline $\operatorname{Age}(\mathrm{yrs})(\mathrm{mean} / \mathrm{SD})$ & \multicolumn{3}{|c|}{$56.06 \pm 11.21$} \\
\hline \multirow{2}{*}{ Gender (n) } & Male & 38 & \\
\hline & Female & 12 & \\
\hline Heart rate $(\mathrm{bpm})($ mean/SD) & \multicolumn{3}{|c|}{$93.08 \pm 11.67$} \\
\hline $\begin{array}{l}\text { Ejection fraction }(\%) \\
(\text { mean/SD) }\end{array}$ & \multicolumn{3}{|c|}{$41.36 \pm 6.23$} \\
\hline \multirow{2}{*}{$\begin{array}{l}\text { Canadian cardiovascular class } \\
\text { (n) }\end{array}$} & $0 \quad 1$ & 2 & 3 \\
\hline & $0 \quad 80$ & 14 & 6 \\
\hline \multirow{3}{*}{ Left ventricular dysfunction (n) } & Mild & 20 & \\
\hline & Moderate & 70 & \\
\hline & Severe & 10 & \\
\hline $\mathrm{Hb}(\%)($ mean/SD $)$ & \multicolumn{3}{|c|}{$13.47 \pm 1.95$} \\
\hline WBC (cells/cumm) (mean/SD) & \multicolumn{3}{|c|}{$8873 \pm 1311.88$} \\
\hline $\operatorname{ESR}(\mathrm{mm} / \mathrm{hr})(\mathrm{mean} / \mathrm{SD})$ & \multicolumn{3}{|c|}{$15.17 \pm 1.58$} \\
\hline Blood sugar $($ gms \%) $($ mean/SD $)$ & \multicolumn{3}{|c|}{$168.6 \pm 64.5303$} \\
\hline Serum Na+(meq/L) (mean/SD) & \multicolumn{3}{|c|}{$139.18 \pm 3.29$} \\
\hline Serum K+(meq/L) (mean/SD) & \multicolumn{3}{|c|}{$4.2 \pm 0.3$} \\
\hline $\begin{array}{l}\text { Alkaline phosphatase (IU/L) } \\
\text { (mean/SD) }\end{array}$ & \multicolumn{3}{|c|}{$74.24+30.81$} \\
\hline
\end{tabular}

The demographic data concerning the patient's age, sex, weight, vitals, hemodynamic and laboratory parameters were statistically assessed at the baseline (Table 1). Maximum numbers of patients (19 patients) were in the age group of $51-60 \mathrm{yrs}$ in this study. According to the distribution of gender, in this study $76 \%$ patients were male and $24 \%$ patients were female. Patients with comorbid medical conditions, 19 patients had hypertension and 19 patients had hypertension as well as diabetes.

Efficacy of ivabradine in reducing the heart rate at the end of 1 month $(86.60 \pm 9.16)$ and at the end of 3 months $(81.82 \pm 8.37)$ when compared with baseline $(93.08 \pm 11.67)$. Reduction in heart rate at the end of 1 and 3 months after taking Ivabradine was statistically significant $(p<0.0001)$. Figure 1 showed graphical representation of reduction in heart rate before and after taking tablet Ivabradine. At the baseline 3 patients were in the CCS class of angina grade IV. After treatment with ivabradine, none of the patients were in the CCS class of angina grade IV at the end of 1 and 3 months. At the baseline 7 patients were in the CCS class of angina grade III. After treatment with study drug, patients with CCS class of angina grade III were 3 patients at the end of 1 month and 1 patient at the end of 3 months. At the baseline 40 patients were in the CCS class of angina grade II. After treatment with study drug, patients with CCS class of angina grade II were 8 patients at the end of 1 month and 9 patients at the end of 3 months (Table 2 and Figure 2). No significant changes were seen in 
hemodynamic parameters. No significant elevations in liver enzymes were found during the treatment period and follow up (Table 3).

\section{Adverse drug reactions}

No patients were withdrawn from the study due to adverse drug reactions during the study period. No serious adverse events were reported in study patients. The most common adverse drug reactions reported were tabulated (Table 4)

\section{DISCUSSION}

Coronary artery disease is the leading cause of morbidity and mortality in world wide ${ }^{1}$.Heart rate is a major determinant of cardiac output, myocardial oxygen demand and coronary blood flow. High resting heart rate has emerged as a simple but relevant risk factor for coronary artery disease and heart failure. Pharmacotherapy for IHD is to reduce the angina attacks by reducing the heart rate. Evidence suggested that many patients maintain a resting $\mathrm{HR} \geq 70 / \mathrm{min}$ despite optimal beta blocker therapy. ${ }^{13}$

Table 2: Improvement in Canadian cardiovascular society class of angina grading.

\begin{tabular}{|ccllll|}
\hline CCS class of angina grade & $\mathbf{1}$ & $\mathbf{2}$ & $\mathbf{3}$ & $\mathbf{4}$ \\
\hline Number & Baseline & 0 & 40 & 7 & 3 \\
\cline { 2 - 6 } of & 1 Month & 39 & 8 & 3 & 0 \\
\cline { 2 - 6 } Patients & 3 Months & 40 & 9 & 1 & 0 \\
\hline
\end{tabular}

Table 3: Laboratory parameters of the study population.

\begin{tabular}{|lllllll|}
\hline & \multicolumn{2}{l}{ Hemodynamic parameters } & & RBS & \multicolumn{2}{l|}{ Liver function tests } \\
& Hb\% & $\begin{array}{l}\text { WBC } \\
\text { Parameters }\end{array}$ & $\begin{array}{l}\text { ESR } \\
(\mathbf{m m} / \mathbf{h r})\end{array}$ & $\begin{array}{l}\text { Total } \\
\text { bilirubin(U/L) }\end{array}$ & $\begin{array}{l}\text { AST } \\
\text { (U/L) }\end{array}$ \\
\hline $\begin{array}{l}\text { At the baseline } \\
\text { (Mean/SD) }\end{array}$ & $\begin{array}{l}13.47 \\
\pm 1.95\end{array}$ & $8873 \pm 1311.88$ & $15.17 \pm 1.58$ & $168.6 \pm 64.5$ & $0.81 \pm 0.28$ & $74.24 \pm 30.81$ \\
\hline $\begin{array}{l}\text { At the end of 1 } \\
\text { month (Mean/SD) }\end{array}$ & $\begin{array}{l}13.72 \pm 2.3 \\
5\end{array}$ & $8806 \pm 1190.63$ & $15.34 \pm 1.15$ & $168.74 \pm 53.9$ & $0.82 \pm 0.11$ & $75.48 \pm 23.64$ \\
\hline P value & 0.318 & 0.364 & 0.307 & 0.967 & 0.719 & 0.644 \\
\hline $\begin{array}{l}\text { At the end of 3 } \\
\text { months (Mean/SD) }\end{array}$ & $\begin{array}{l}13.76 \pm 1.8 \\
8\end{array}$ & $8782.5 \pm 1218.9$ & $15.46 \pm 1.75$ & $169.62 \pm 57.7$ & $0.84 \pm 0.11$ & $78.3 \pm 21.13$ \\
\hline P value & 0.196 & 0.359 & 0.164 & 0.766 & 0.419 & 0.329 \\
\hline
\end{tabular}

Table 4: The number and percentage of adverse drug reactions reported during the study period.

\begin{tabular}{|ll|}
\hline $\begin{array}{l}\text { Adverse drug } \\
\text { reactions }\end{array}$ & No. of patients $\mathbf{n}(\%)$ \\
\hline Bradyarrythmia & $2(4 \%)$ \\
\hline QT prolongation & $1(2 \%)$ \\
\hline Blurring of vision & $1(2 \%)$ \\
\hline Photopsia & $1(2 \%)$ \\
\hline
\end{tabular}

Conventional anti-anginal drugs like nitrates, $\mathrm{CCB}$ and $\mathrm{K}$ + channel openers could interact with beta-blockers and in addition they have negative inotropic activity. So we need a better agent which will reduce the heart rate without having negative inotropic activity and other system involvement. In recent years, a new drug to reduce $H R$, Ivabradine has been introduced for clinical practice by Euro Society of Cardiology .According to the results of the initiative trial which compared Ivabradine with atenolol over 4 months in 939 patients with stable angina pectoris and documented coronary artery disease, Ivabradine showed non-inferiority in reducing the total exercise duration and exercise performance and improved performance. $^{14}$

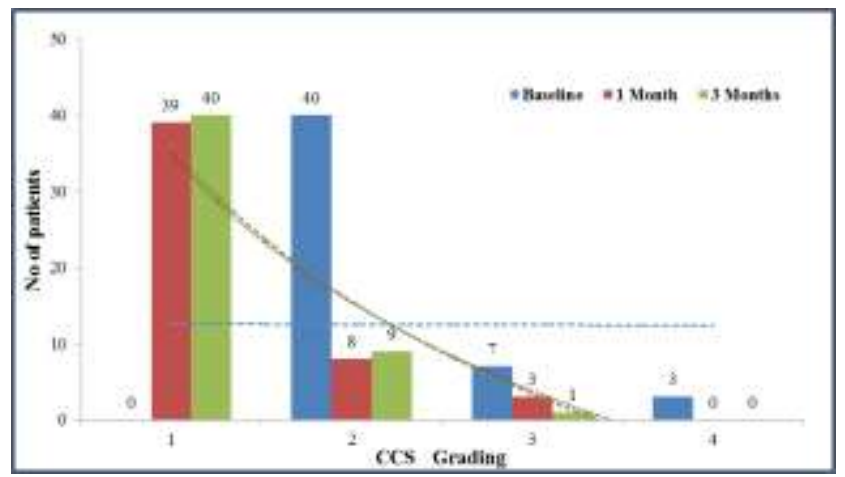

Figure 1: Improvement in Canadian cardiovascular society grading of angina pectoris.

Figure 2 showed Graphical representation of improvement of Canadian cardiovascular society grading (CCS) of angina at the end of 1 and 3 months compared with baseline.

Blue dotted line in the above graph is the logarithmic trend line of baseline CCS compared with $1^{\text {st }}$ month (red dotted line) and $3^{\text {rd }}$ month (green dotted line) logarithmic trend lines. 
Based on the comparison of logarithmic trend line at the baseline with 1 and 3 months Ivabradine showed improvement in CCS class of grade of angina.

The mean age of the subjects in our study was $56.06 \pm 11.21$ (Table 1). It clearly showed that ageing is an important non-modifiable risk factor for chronic stable ischemic heart disease. Similarly, a prospective, followup study conducted in Finland concluded that in both sexes the CHD risk was largest in the oldest age (50$69 \mathrm{yrs}$ ) group ${ }^{15}$. According to our study, the majority of the study subjects were male patients $76 \%$ and males in the community were more prone for ischemic heart disease than females. In one study conducted by Peters and Woodwards showed that over a median follow-up of 6.7 years, 5695 IHD events were documented. Based on the major risk factors, the age-adjusted prevalence was higher in males than females. The hazard ratio for IHD, comparing men with women, was 1.88 (95\% CI 1.542.29) in Asia and 2.14 (95\% CI 1.97-2.33) in Australian and New Zealand. ${ }^{16}$

In our study, significant reduction in heart rate was reported in patients with CHD during the treatment period was $81.82 \pm 8.37$ (Figure 2) compared with baseline $93.08 \pm 11.67$ and the $\mathrm{p}$ value was significant $(<0.001)$. Similarly a study conducted by Jochmann $N$ and Franziska et al found a significant decrease in heart rate, both 4 hours after the intake of $7.5 \mathrm{mg}$ of ivabradine (median -8 [interquartile range (IQR) - 14 to -4 ] bpm) and after 4 weeks of twice daily intake (median - 10 $[\mathrm{IQR}-17$ to -5$] \mathrm{bpm})(\mathrm{p}<0.05) .{ }^{17}$ Thus for preventing and treating the further anginal attacks reducing the heart rate is the important key factor without producing negative inotropic and lusitropic effect. Our study drug by acting on the novel specific and selective blocking of if current present in the SA node of the right atrial chamber showed significant reduction in heart rate (Figure 2).

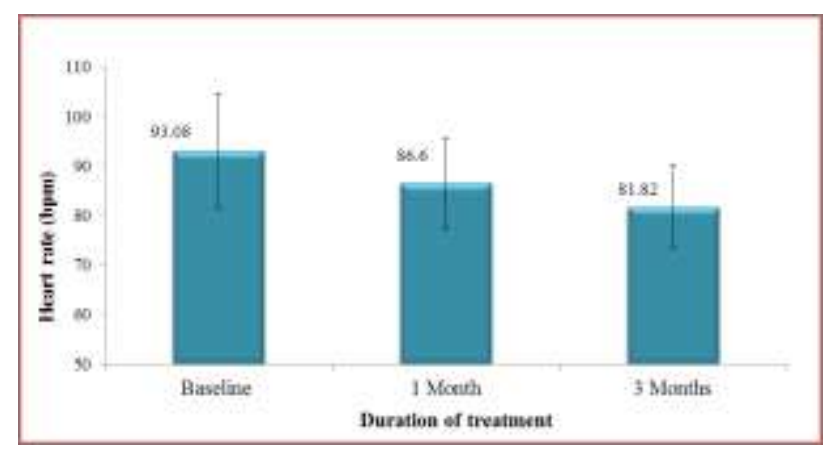

\section{Figure 2: Reduction in heart rate at the end of 1 and 3 months compared with baseline.}

Graphical representation of reduction in heart rate before and after taking tablet Ivabradine. Reduction in heart rate from baseline $(93.08 \pm 11.67)$ to end of the 1 st month $(86.60 \pm 9.16)$ and at the end of 3rd month $(81.82 \pm 8.37)$ (Figure 2).
In this study, treatment with ivabradine improved the CCS class of angina grading compared with baseline. Those patients at the grade III and IV at baseline were moved to grade I and II over a period of 3 months. At the baseline 3 patients were in the CCS class of angina grade IV. After treatment with ivabradine, none of the patients in the CCS class of angina grade IV at the end of 1 and 3 months. At the baseline 7 patients were in the CCS class of angina grade III. After treatment with study drug, patients with CCS class of angina grade III were 3 patients at the end of 1 month and 1 patient at the end of 3 months. At the baseline 40 patients were in the CCS class of angina grade II. After treatment with study drug, patients with CCS class of angina grade II were 8 patients at the end of 1 month and 9 patients at the end of 3 months (Table 2 and Figure 2). Similarly, in a post hoc analysis of beautiful Trial suggested that the effect of adding Ivabradine to the conventional beta-blocker (90\%) improved the CCS class of grade of angina. ${ }^{18}$

In our study, ivabradine did not influence the blood pressure. The routine hematological and biochemical evaluations did not show any significant difference (Table 3 ) in the pre-post values $(p>0.05)$. A study conducted by Aditi Chaturvedi, Yogendra Singh et al. showed that Hb, LFTs, RFTs, serum electrolytes and RBS done at baseline and after 8 weeks of use of the Ivabradine did not show any significant difference $\mathrm{p}>0.05$ (using the paired " $t$ " test). ${ }^{19}$ In our study, Ivabradine related adverse drug reactions reported were bradyarrythmia, QT prolongation and visual disturbances like blurring of vision and photopsia (Table 4). Study conducted by Borer et al, Tardif et al and Ruzyllo et al. ${ }^{20-}$ ${ }^{22}$ These reactions were considered to be mild and abated on its own after treatment period. But no patients were withdrawn from the study due to adverse drug reactions and no patients experienced serious adverse drug reactions.

Efficacy of Ivabradine by assessing the reduction in heart rate, CCS class of anginal grading improvement in patients with chronic stable angina were the strength of this study. Small sample size and lack of long term follow up were the limitations of this study. Thus the present study showed that Ivabradine causes reduction in HR and improvement in CCS class of angina grading. Therefore the study drug, Ivabradine is safe and effective when given along with atenolol in treating chronic stable ischemic heart disease. Further studies are needed with larger sample size and long term follow up.

\section{CONCLUSION}

Based on the results of our study, we conclude that Ivabradine is safe and effective in preventing and treating further anginal attacks in patients with chronic stable ischemic heart disease already on atenolol therapy. 


\section{ACKNOWLEDGEMENTS}

We would like to thank Dr. J. Ezhil Ramya M.D, Tirunelveli medical college, Tirunelveli for allowing us to conduct this study.

Funding: No funding sources Conflict of interest: None declared

Ethical approval: The study was approved by the Institutional Ethics Committee

\section{REFERENCES}

1. Jackson JM. Ivabradine - a novel treatment for chronic stable angina. Drugs in Context: e212225. DOI: $10.7573 /$ dic. 212225 .

2. Cassar A, Holmes DR, Rihal CS. Chronic coronary artery disease: diagnosis and management. Mayo Clin Proc. 2009;84(12):1130-46.

3. Morrow DA, Boden WE. Stable Ischemic Heart Disease. Braunwald's Heart Disease, A textbook of cardiovascular Medicine. Philadelphia Elsevier. 2012. pp. 1210-1211.

4. Deedwania P. Selective and specific inhibition of if with ivabradine for the treatment of coronary artery disease or heart failure, drugs. Springer. 2013;73:1569-86.

5. ZakyH, Elzein H, Alsheikh-Ali AA, Al-Mulla A. Short-term effects of ivabradine in patients with chronic stable ischemic heart disease. Heart Views 2013;14:53-55.

6. Pflieger M. Management of stable coronary artery disease. American Family Physician. 2011;83(7):820-5 .

7. Hjemdahl P, Eriksson SV, Held C, Forslund L, Näsman $P$, Rehnqvist N. Favourable long term prognosis instable angina pectoris: An extended follow up of the angina prognosis study in Stockholm (APSIS). Heart. 2006;92:177-82.

8. Ferrari R, Campo G, Gardini E, Pasanisi G, Ceconi C. Specific and selective If inhibition: expected clinical benefits from pure heart rate reduction in coronary patients. European Heart Journal Supplements. 2005;7(Supplement H):H16-21.

9. Shattock M, Camm AJ. Pure Heart Rate Reduction: The If Channels From Discovery to Therapeutic Target. Br J Cardiol. 2006;13(1):27-35.

10. Michel T. Treatment of Myocardial Ischemia and Hypertension. Goodman and Gilman's The Pharmacological Basis Of Therapeutics, New York: McGraw-Hill. 2011;27:772-3.
11. Kones R. Recent advances in the management of chronic stable angina II. Anti-ischemic therapy, options for refractory angina, risk factor reduction and revascularization. Vascular Health and Risk Management. 2010;6:749-74.

12. Michel T, Hoffman BB. Treatment of Myocardial Ischemia and Hypertension .Goodman and Gilman's The Pharmacological basis of therapeutics, New York: McGraw-Hill. 2011;27:757-773.

13. Di Franco A, Sarullo FM, Salerno Y. Beta-blockers and ivabradine in chronic heart failure: from clinical trials to clinical practice. Am J Cardiovasc Drugs. 2014;14(2):101-10.

14. Sulfi S, Timmis AD. Ivabradine - the first selective sinus node If channel inhibitor in the treatment of stable angina. Int J Clin Pract. 2006;60(2):222-8.

15. Jousilahti P, Vartiainen E, Tuomilehto J, Puska P. Sex, age, cardiovascular risk factors, and coronary heart disease: a prospective follow-up study of 14 786 middle-aged men and women in Finland. Circulation. 1999;99(9):1165-72.

16. Peters SAE, Woodward M, Lam TH, Suh II. Sex disparities in risk and risk factors for ischemic heart disease in the Asia-Pacific region. Europ Jou Preven Cardiolo. 2014;21(5):639-46.

17. Jochmann N. Effect of ivabradine-induced heart rate reduction on flow-mediated dilation measured with high-sensitivity ultrasound in patients with stable coronary heart disease. Cardiovascular ultrasound. 2014;12(5):1-6.

18. Lopez E, Lopez J. beta blockers. Pharmacological Treatment of Chronic Stable Angina Pectoris. Current cardiovascular therapy. Springer international New York. 2015. pp.57-70.

19. Chaturvedi A, Singh Y. Comparison of the efficacy and tolerability of ivabradine and ranolazine in patients of chronic stable angina pectoris. Journal of pharmacology and pharmacotherapeutics. 2013;4(1):33-8.

20. Borer JS, Jaillon P. Antianginal and antiischemic effects of ivabradine, an I(f) inhibitor, in stable angina: a randomized, double-blind, multicentered, placebo-controlled trial. Circulation. 2003;107(6):817-23.

21. Marquis-Gravel G, Tardif JC. Ivabradine: the evidence of its therapeutic impact in angina. Core Evid. 2008;3(1):1-12.

22. Hochman JS, Ruzyllo W. Coronary Intervention for Persistent Occlusion after Myocardial Infarction. N Engl J Med. 2006;355(23):2395-407.

Cite this article as: Elavarasi P, Ezhil RJ, Vasanth S. Efficacy and safety of ivabradine as an add-on to atenolol in patients with chronic stable ischemic heart disease. Int J Basic Clin Pharmacol 2016;5:2546-51. 